\title{
Cross-sectional and Longitudinal Evaluation of Bone Mass in Children and Young Adults with Juvenile Idiopathic Arthritis: The Role of Bone Mass Determinants in a Large Cohort of Patients
}

\author{
STEFANO STAGI, LAURA MASI, SERENA CAPANNINI, ROLANDO CIMAZ, GIULIA TONINI, \\ MARCO MATUCCI-CERINIC, MAURIZIO de MARTINO, and FERNANDA FALCINI
}

\begin{abstract}
Objective. To assess the prevalence of reduced spine bone mineral apparent density (BMAD), and to identify the main predictors of reduced spine BMAD in a cross-sectional and longitudinal evaluation of the same large cohort of patients with juvenile idiopathic arthritis (JIA). There are few prospective data on bone mass evaluation in a large number of patients with JIA, and with enthesitis-related arthritis onset.

Methods. Two hundred nineteen patients with JIA (median age $8.7 \mathrm{yrs}$, range 6.1-13.1 yrs; 104 oligoarticular JIA, 61 polyarticular, 20 systemic, and 34 enthesitis-related arthritis onset) were retrospectively evaluated. A dual-energy x-ray absorptiometry (DEXA) scan at the lumbar spine was performed in all subjects. Of these, 89 consecutive patients were followed up randomly and longitudinally with a second and a third DEXA evaluation. The data obtained were compared with 80 age-matched and sex-matched healthy subjects.
\end{abstract}

Results. At the first DEXA, patients with JIA showed a reduced spine BMAD standard deviation score (SDS) in comparison to controls $(\mathrm{p}<0.001)$. These results were confirmed when the subjects were divided into JIA subtypes $(\mathrm{p}<0.005)$ with the exception of enthesitis-related arthritis onset. Spine BMAD SDS significantly correlated with JIA onset type $(\mathrm{p}<0.01)$, age at JIA onset $(\mathrm{p}<$ $0.005)$, and flares $(\mathrm{p}=0.008)$. The longitudinal evaluation showed that spine BMAD SDS did not significantly improve at the followup in comparison to controls, in all subsets with JIA except for systemic onset $(\mathrm{p}<0.05)$. Spine BMAD correlated with sex $(\mathrm{p}<0.01)$, systemic corticosteroid exposure $(\mathrm{p}<0.01)$, the number of intraarticular corticosteroid injections $(\mathrm{p}<0.01)$, the interval from last steroid injection $(\mathrm{p}<0.05)$, erythrocyte sedimentation rate $(\mathrm{p}<0.005)$, and C-reactive protein levels $(\mathrm{p}<0.005)$

Conclusion. Patients with JIA have a low bone mass and, after a first increase due to therapy, do not reach a healthy condition over time despite our current more effective drugs. These patients have a high risk of osteoporosis in early adulthood. To reduce the risk and improve the bone mass, close monitoring of bone mineral density, better control of disease activity, physical activity, and intake of calcium and vitamin D are recommended. In patients with osteoporosis, therapeutic approaches including bisphosphonates should be considered. (First Release June 1 2010; J Rheumatol 2010; 37:1935-43; doi:10.3899/jrheum.091241)

Key Indexing Terms:

BONE MASS

JUVENILE IDIOPATHIC ARTHRITIS

From the Pediatric Unit, Villamarina Hospital, Piombino, Livorno, Department of BioMedicine, Section of Rheumatology, Transition Clinic; Department of Internal Medicine, Endocrinology Unit; Department of Pediatrics, Anna Meyer Children's Hospital; and Department of Statistics, University of Florence, Florence, Italy.

S. Stagi, MD, Pediatric Unit, Villamarina Hospital; L. Masi, MD, Department of Internal Medicine, Endocrinology Unit, University of Florence; S. Capannini, MD; M. Matucci-Cerinic, MD; F. Falcini, MD, Department of BioMedicine, Section of Rheumatology, Transition Clinic, University of Florence; R. Cimaz, MD; M. de Martino, MD, Department of Pediatrics, Anna Meyer Children's Hospital, University of Florence; G. Tonini, MD, Department of Statistics, University of Florence.

Address correspondence to Dr. F. Falcini, Department of Biomedicine, Section of Rheumatology, Transition Clinic, University of Florence, Viale Pieraccini 18, 50139 Florence, Italy. E-mail:falcini@unifi.it

Accepted for publication March 4, 2010.

\section{BONE MINERAL DENSITY OSTEOPOROSIS}

In all subgroups of juvenile idiopathic arthritis (JIA), either a pathologic loss or lack of increase in bone mass is reported in a high percentage of children and young adults despite the new therapeutic approaches $1,2,3,4,5$. The decrease in bone mass is multifactorial in origin ${ }^{6}$ and correlates with the duration of active disease and the number of affected joints ${ }^{7}$.

Chronic inflammation may result in periarticular and generalized bone $\operatorname{loss}^{8,9}$. Cytokines and growth factors produced by synovial tissue increase osteoclast formation, activity, and/or survival, and thus enhance bone resorption ${ }^{10}$. In addition, limited physical activity, and reduced muscle strength acting on bones, represent additional risk factors for osteoporosis $^{11,12}$. 
Further, drugs, especially corticosteroids, lower bone mineral density (BMD) 2,3,4,5,13. Methotrexate (MTX) induces osteopenia in childhood malignancies ${ }^{14}$, while in patients with JIA it has proven to increase bone mass, reducing the rate and the duration of inflammation ${ }^{15}$. A recent study in children receiving tumor necrosis factor- $\alpha$ (TNF- $\alpha$ ) inhibitors revealed increased BMD at 12 months of treatment, due to successful control of inflammation and specific interaction with bone metabolism ${ }^{16}$.

This raises great concern because poor bone quality, osteopenia, and osteoporosis may lead to reduced bone strength and increased fracture risk, in particular vertebral collapse after minor trauma ${ }^{17,18}$, worsening the prognosis of the disease.

Recently, a detailed characterization of physiological interaction between muscle and bone that persists after the disease remission and represents an important outcome variable has been reported in patients with $\mathrm{JIA}^{7}$. Additionally, it has also been observed that the frequency of low bone mass is higher in young adults with a history of early-onset JIA ${ }^{19}$.

Although cross-sectional studies on BMD in JIA are numerous and frequently show different and conflicting results $2,5,13,20,21,22,23,24$, there are few prospective data on bone mass evaluation in a large number of patients with JIA $^{7,19,25}$. Further, there are few reports regarding bone mass assessment in subjects with enthesitis-related arthritis onset $^{20}$.

Our purpose was to evaluate through a cross-sectional and longitudinal study the spinal bone mineral apparent density (BMAD) in a large cohort of patients with JIA, and to identify the main predictors of reduced bone mass.

\section{MATERIALS AND METHODS}

A retrospective cross-sectional and longitudinal case control study was performed. Consecutive patients were recruited from the Department of BioMedicine, Section of Rheumatology, Transition Clinic of The University of Florence, and from the Pediatric Rheumatology Unit of Anna Meyer Children's Hospital between January 2001 and December 2008.

The study protocol was approved by our Hospital Ethics Committees and informed consent was obtained from all patients and/or parents.

Study population and design. Two hundred nineteen patients fulfilling the criteria $^{26}$ for JIA were evaluated (155 females, 64 males; median age at evaluation $8.7 \mathrm{yrs}$, range 6.1-13.1 yrs). One hundred four had oligoarticular JIA; 61 polyarticular, rheumatoid factor-negative; 20 systemic; and 34 enthesitis-related arthritis onset. Of these, 89 patients (64 females, 25 males; 43 oligoarticular, 24 polyarticular, 10 systemic, and 12 enthesitisrelated arthritis) were followed longitudinally with a second (median age 11.6 yrs, range 8.3-15.6), and a third (median age 13.3 yrs, range 9.8-17.8) dual-energy x-ray absorptiometry (DEXA) evaluation. Of the oligoarticular patients, 30 (29\%) had extended arthritis at the first DEXA evaluation, while an additional $13(30 \%)$ became extended at the second and third evaluations.

The onset of disease was defined as the date on which arthritis and/or systemic features were documented by a pediatric rheumatologist. The disease subtype was defined according to reported definition ${ }^{26}$. Active JIA was defined as the presence of systemic features (spiking fever, rash), active joint inflammation (swollen/tender/limited joint), and erythrocyte sedimentation rate $(\mathrm{ESR})>20 \mathrm{~mm} / \mathrm{h}$ in systemic onset, more than 5 joints active and ESR $>20 \mathrm{~mm} / \mathrm{h}$ in polyarticular onset, and 1 to 4 joints with active inflammation in oligoarticular onset, within the first 6 months from disease onset. Baseline clinical assessment and laboratory evaluation were performed longitudinally to report the disease course and flares.

For each patient, clinical and demographic data including JIA type, onset, age at diagnosis, disease course, therapy, height, pubertal stage, weight, body mass index (BMI), and family history for osteoporosis were recorded from medical charts.

Study and laboratory methods. Height was measured using a wall-mounted stadiometer, and weight was measured to the nearest $0.1 \mathrm{~kg}$. BMI was calculated as weight divided by height squared $\left(\mathrm{kg} / \mathrm{m}^{2}\right)$. Age-related reference values of height and BMI were obtained from a wide sample of Italian children $^{27}$

Height and BMI were normalized for chronological age by conversion to standard deviation scores (SDS). SDS were calculated according to the following formula: patient value minus mean of age-related reference value/standard deviation of the age-related reference value.

Pubertal staging was carried out according to Tanner and Whitehouse's criteria ${ }^{28}$. The timing of puberty was assessed and the comparison between the healthy population and patients with JIA was as described ${ }^{29}$. In particular, the age of pubertal onset was defined as the age at durable Tanner B2 stage for females or a testicular volume more than $4 \mathrm{ml}$ for males (G2). The age at which this occurred was taken as midway between the previous clinic visit when the child was still prepubertal and the clinic visit when the child was G2/B2. Duration of puberty was taken as time from G2/B2 to G4/B4. Age at G4/B4 was assessed similarly, by taking the age midway between the previous clinic visit when the child was G3/B3 and the clinic visit when the child was G4/B4.

BMD $\left(\mathrm{g} / \mathrm{cm}^{2}\right)$ was measured at the lumbar spine (L2-L4) by DEXA using a strict protocol with the same instrument (Delphi-A System, Hologic Inc., Waltham, MA, USA).

BMD was expressed as Z scores (i.e., the difference between the patient's score and the normal score for that age divided by the SD of the healthy controls). Average BMD values for L2-L4 were used for calculations.

The DEXA instrument's software calculates BMD by dividing the bone mineral content by the area of the projection surface of bones (areal BMD; $\mathrm{g} / \mathrm{cm}^{2}$ ). This does not take into account the actual bone volume, which is strictly related to body size (weight and height), a particularly important aspect when evaluating a growing skeleton. Different methods of correction have been proposed for pathologies where a smaller than normal body size may be present ${ }^{20}$.

For estimation of the respective volumetric density usually called bone mineral apparent density (BMAD), the following formula of Kröger, $e t a l^{30}$ was used:

$$
\mathrm{BMAD}=\mathrm{BMD}_{\mathrm{L} 2-\mathrm{L} 4} \times[4 /(\pi \times \text { width })], \text { expressed in }\left[\mathrm{g} / \mathrm{cm}^{3}\right]
$$

Bone width was the real mean width of these vertebrae. It was calculated from the dimensions that were manually read with a ruler from the radiograph of the spine that was included in the printout of the results of each measurement. If A2, A3, and A4 are the real projected areas of the respective vertebrae, $\mathrm{h}$ is their depicted total height and $\mathrm{b}$ their depicted mean width, then

$$
\text { bone width }=\sqrt{\left[\mathrm{A}_{2}+\mathrm{A}_{3}+\mathrm{A}_{4} / \mathrm{h}\right] \times \mathrm{b}}
$$

Each measurement was taken along the vertebral body at 3 locations (upper, middle, and lower part of the vertebra) by the same researcher, and the mean from these measurements was used; the intraobserver coefficient of variation was $1 \%$.

The Kröger model was validated by in vivo volumetric data obtained from magnetic resonance imaging of the lumbar vertebrae ${ }^{31}$.

Patients' BMAD was expressed as Z scores to facilitate comparisons between patients with JIA and healthy controls. Quality control was performed regularly using a phantom to ensure the reliability of the densitometer. All BMD measurements were performed by the same operator and the same DEXA instrument using a standardized protocol of measurement. 
Clinical assessment of disease activity. Clinical disease activity was determined on the basis of the core set criteria for JIA ${ }^{32}$. Documentation of patient data included medical history and physical examination, in particular the number of active joints (swelling, heat, tenderness, or pain on motion), the number of joints with limited range of motion, physician's global assessment of disease activity, parent's/patient's assessment of overall well-being [visual analog scale as part of the Childhood Health Assessment Questionnaire (CHAQ)], and functional ability (disability as measured in 8 domains by the CHAQ).

The leukocyte count, absolute neutrophil count, red blood cell count, platelet count (all cells $/ \mu \mathrm{l}), \mathrm{ESR}(\mathrm{mm} / \mathrm{h})$, and C-reactive protein (CRP) level (mg/dl) were measured as serum markers of inflammation.

Patients were categorized as having active disease (joint active or sign of systemic disease) or being in clinical remission for at least 6 consecutive months, including absence of morning stiffness, no active arthritis, and ESR $<20 \mathrm{~mm} / \mathrm{h}$, independent of medication in systemic and polyarticular patients. Relapse was defined according to the preliminary definition of disease flare in JIA ${ }^{33}$

Type of treatment. During the cross-sectional and longitudinal evaluations, to define the effect of these determinants on spine BMAD values, we considered the following therapies: nonsteroidal antiinflammatory drugs (NSAID), sulfasalazine, systemic and/or intraarticular corticosteroids, MTX, and TNF- $\alpha$ inhibitors.

For all drugs, the period of administration was also recorded. In particular, in patients who had taken systemic corticosteroids, the duration of corticosteroid exposure was obtained from the clinic, hospital, and emergency department records, and the data were summed to yield a cumulative dose $(\mathrm{mg} / \mathrm{kg})$ from the time of diagnosis to the time of the first, second, and third DEXA. Moreover, the number of glucocorticoid intraarticular injections and the interval between the injection and DEXA assessment were noted. For all systemic corticosteroids we converted the steroid dosage to a common steroid equivalency. However, for intraarticular injections we simply counted the number of courses.

Laboratory and functional assessments. Hemoglobin, white blood cell, platelet count, CRP (Westergren), CRP, serum creatinine, calcium, phosphate, and urinary calcium excretion corrected for creatinine were measured using standard tests.

Healthy controls. At the first DEXA, the control group included 80 healthy age-matched and sex-matched subjects (58 females, 22 males; median age at DEXA evaluation 8.6 yrs, range 6.0-13.3) seen at our units for noninflammatory musculoskeletal complaints.

At the second and third DEXA evaluations, we selected 2 age-matched and sex-matched control groups with the same characteristics (second DEXA: 82 subjects, 59 females, 23 males, median age 11.4 yrs, range 8.2-15.4; third DEXA: 78 subjects, 56 females, 22 males, median age 13.1 yrs, range 9.7-17.9).

For all controls, exclusion criteria were chronic diseases or medication exposures known to affect bone mass (e.g., renal or hepatic disease, endocrine disorders, or a history of glucocorticoid or anticonvulsant therapy). All subjects were evaluated at the time of routine followup visits, and parental informed consent was obtained.

Statistical analysis. Statistical analyses were performed using SPSSX (SPSS Inc., Chicago, IL, USA). Clinical variables considered relevant for the study were sex, subtype of JIA, spine BMAD SDS, age at JIA onset, disease duration at DEXA examination, time from JIA diagnosis to DEXA examinations (in years), flares between JIA onset and DEXA examination or between 2 DEXA examinations, disease activity at DEXA examinations (physician's global score, joint score), BMI SDS, height SDS, age of puberty onset, pubertal stage at DEXA examination, ESR, CRP, hemoglobin, type of treatment [yes/no: NSAID, sulfasalazine, systemic and/or intraarticular corticosteroids, MTX, TNF- $\alpha$ inhibitors], number of intraarticular steroid injections, interval from last steroid injection to DEXA examination, and systemic steroid dosage.

Summaries of continuous variables are given as mean \pm SD or median and range, depending on whether the data were normally distributed or not. Differences between patient groups and controls were assessed using the Student $\mathrm{t}$ test and Mann-Whitney $\mathrm{U}$ test, depending on the distribution of the analyzed variable. The chi-square test and Fisher's exact test were used as appropriate to examine associations between dichotomous variables Intergroup comparisons for measurements were conducted using ANOVA or repeated-measures analysis of covariance (ANCOVA), as appropriate.

Spearman's (rank) correlation test was used to determine the correlation coefficients. A multiple stepwise regression was used to determine the variables that may correlate independently with lumbar spine BMAD SDS values. $\mathrm{P}$ values $<0.05$ were considered statistically significant.

\section{RESULTS}

The main demographic, clinical, and laboratory characteristics of patients with JIA and controls are summarized in Tables 1 and 2 .

At the first DEXA, all patients with JIA showed reduced spine BMAD SDS values in comparison to controls $(-0.78$ \pm 1.00 vs $0.02 \pm 0.94 ; \mathrm{p}<0.001)$. These results were still observed when the subjects were divided into the main 3 JIA subtypes (oligoarticular: $-0.41 \pm 0.7, \mathrm{p}<0.005$; polyarticular: $-0.63 \pm 0.8, \mathrm{p}<0.0001$; systemic: $-1.41 \pm 0.7$, $\mathrm{p}<0.0001)$. In contrast, subjects with enthesitis-related arthritis did not show any difference in comparison to controls $[-0.09 \pm 0.5 ; \mathrm{p}=$ nonsignificant $(\mathrm{NS})$; Figure 1]. At the time of the first DEXA, disease duration was not significantly different among the subgroups of patients (Table 1).

Table 1. Data on 219 patients with JIA and 80 controls at the first DEXA evaluation.

\begin{tabular}{lccc}
\hline Characteristic & JIA & Controls (n) & $\mathrm{p}$ \\
\hline No. of patients at first DEXA (F:M) & $155: 64$ & $56: 24$ & $\mathrm{NS}$ \\
Age at first DEXA, yrs \pm SD & $8.5 \pm 3.3$ & $8.5 \pm 4.0$ & $\mathrm{NS}$ \\
Type of JIA onset (\%) & & & \\
$\quad$ Oligoarticular & $105(48)$ & & \\
Polyarticular & $61(28)$ & - & - \\
$\quad$ Systemic & $20(9)$ & & \\
Enthesitis-related arthritis & $34(15)$ & & \\
Age at diagnosis, yrs \pm SD & $5.0 \pm 3.8$ & & \\
Oligoarticular & $4.7 \pm 2.7$ & & \\
Polyarticular & $5.7 \pm 3.7$ & - & - \\
Systemic & $4.0 \pm 3.4$ & & \\
Enthesitis-related arthritis & $8.5 \pm 2.7$ & & \\
Disease duration, yrs \pm SD & $3.5 \pm 3.0$ & & \\
$\quad$ Oligoarticular & $3.6 \pm 2.9$ & & \\
Polyarticular & $3.1 \pm 3.2$ & - & \\
Systemic & $3.2 \pm 3.1$ & & \\
$\quad$ Enthesitis-related arthritis & $3.1 \pm 2.6$ & & \\
Height (SDS) & $-0.6 \pm 1.1$ & $-0.1 \pm 0.9$ & $<0.05$ \\
BMI (SDS) & $-0.3 \pm 0.8$ & $0.1 \pm 0.7$ & $\mathrm{NS}$ \\
BMAD L2-L4 SDS & & & \\
Oligoarticular & $-0.41 \pm 0.7$ & $0.02 \pm 0.9$ & $<0.005$ \\
Polyarticular & $-0.63 \pm 0.8$ & $0.02 \pm 0.9$ & $<0.001$ \\
Systemic & $-1.41 \pm 0.7$ & $0.02 \pm 0.9$ & $<0.001$ \\
Enthesitis-related arthritis & $-0.09 \pm 0.5$ & $0.02 \pm 0.9$ & $\mathrm{NS}$ \\
Total & $-0.78 \pm 1.00$ & $0.02 \pm 0.9$ & $\mathrm{NS}$ \\
\hline & & & \\
\hline
\end{tabular}

JIA: juvenile idiopathic arthritis; DEXA: dual energy x-ray absorptiometry; SDS: standard deviation scores; BMAD: bone mineral apparent density; BMI: body mass index; NS: nonsignificant. 
Table 2. Data on 89 patients with JIA longitudinally evaluated and their respective control groups, at second and third DEXA evaluation.

\begin{tabular}{|c|c|c|c|}
\hline Characteristic & JIA & Controls (n) & $\mathrm{p}$ \\
\hline \multicolumn{2}{|c|}{ No. of patients at second DEXA (F:M) $64: 25$} & $59: 23$ & NS \\
\hline Age at second DEXA, yrs \pm SD & $12.1 \pm 2.9$ & $11.8 \pm 3.2$ & NS \\
\hline \multicolumn{4}{|c|}{ Type of JIA onset at second and third DEXA (\%) } \\
\hline Oligoarticular & $43(48)$ & & \\
\hline Polyarticular & $24(27)$ & - & - \\
\hline Systemic & $10(11)$ & & \\
\hline Enthesitis-related arthritis & $12(14)$ & & \\
\hline \multicolumn{4}{|l|}{ Disease duration at second DEXA, } \\
\hline yrs $\pm \mathrm{SD}$ & $7.1 \pm 3.5$ & & \\
\hline Oligoarticular & $7.4 \pm 3.6$ & & \\
\hline Polyarticular & $6.5 \pm 3.3$ & - & - \\
\hline Systemic & $7.1 \pm 3.6$ & & \\
\hline Enthesitis-related arthritis & $5.3 \pm 3.1$ & & \\
\hline No. of disease flares at second DEXA & $1.2 \pm 1.3$ & & \\
\hline \multicolumn{4}{|c|}{ BMAD L2-L4 SDS at second DEXA } \\
\hline Oligoarticular & $-0.52 \pm 0.8$ & $0.10 \pm 0.8$ & $<0.005$ \\
\hline Polyarticular & $-0.60 \pm 0.8$ & $0.10 \pm 0.8$ & $<0.001$ \\
\hline Systemic & $-1.19 \pm 0.5$ & $0.10 \pm 0.8$ & $<0.001$ \\
\hline Enthesitis-related arthritis & $-0.10 \pm 0.8$ & $0.10 \pm 0.8$ & NS \\
\hline No. of patients at third DEXA (F:M) & $64: 25$ & $56: 22$ & NS \\
\hline Age at third DEXA, yrs \pm SD & $13.8 \pm 3.2$ & $13.4 \pm 3.8$ & NS \\
\hline \multicolumn{4}{|l|}{ Disease duration at third DEXA, } \\
\hline yrs \pm SD & $8.3 \pm 3.4$ & & \\
\hline Oligoarticular & $9.1 \pm 3.6$ & & \\
\hline Polyarticular & $8.3 \pm 3.7$ & - & - \\
\hline Systemic & $9.0 \pm 3.3$ & & \\
\hline Enthesitis-related arthritis & $7.4 \pm 3.5$ & & \\
\hline No. of disease flares at the third DEXA & A $1.5 \pm 1.2$ & & \\
\hline \multicolumn{4}{|c|}{ BMAD L2-L4 SDS at third DEXA } \\
\hline Oligoarticular & $-0.46 \pm 0.9$ & $0.07 \pm 0.9$ & $<0.005$ \\
\hline Polyarticular & $-0.62 \pm 0.7$ & $0.07 \pm 0.9$ & $<0.001$ \\
\hline Systemic & $-1.11 \pm 0.8$ & $0.07 \pm 0.9$ & $<0.001$ \\
\hline Enthesitis-related arthritis & $-0.20 \pm 0.9$ & $0.07 \pm 0.9$ & NS \\
\hline
\end{tabular}

JIA: juvenile idiopathic arthritis; DEXA: dual energy x-ray absorptiometry; SDS: standard deviation scores; BMAD: bone mineral apparent density; BMI: body mass index; NS: nonsignificant.

Among the JIA groups, spine BMAD SDS significantly correlated with the type of onset. In particular, patients with systemic arthritis had a lower spine BMAD SDS with respect to both oligoarticular $(\mathrm{p}<0.01)$ and enthesitis-related arthritis $(\mathrm{p}<0.005)$, while patients with polyarticular onset had a significantly lower spine BMAD SDS with respect to children with enthesitis-related arthritis ( $\mathrm{p}<$ 0.01). However, there was no statistical difference between patients with oligoarticular JIA and those with enthesitisrelated arthritis.

In patients with JIA, spine BMAD SDS showed a significant correlation with the age at onset $(r=0.45 ; \mathrm{p}<0.005)$, and flares $(\mathrm{r}=-0.34 ; \mathrm{p}=0.008)$. Disease activity proved a significant correlation with BMI SDS $(r=-0.46 ; \mathrm{p}<0.001)$ and height SDS $(r=-0.36 ; \mathrm{p}=0.004)$.

At first DEXA, $42 \%$ of our patients with JIA were taking NSAID. MTX was prescribed to $49.3 \%$ and TNF- $\alpha$ inhibitors to $18 \%$ of patients, while $34 \%$ took sulfasalazine.
High-dose systemic or oral glucocorticoids were given only to patients with systemic JIA onset $(\mathrm{p}<0.001)$, while the dose administered in the other subtypes of the disease was low, without statistically significant differences in the different groups.

Evaluating the different drugs, a significant correlation among spine BMAD values, systemic corticosteroid exposure, number of intraarticular corticosteroid injections, and TNF- $\alpha$ inhibitors was detected (respectively: $\mathrm{r}=-0.45, \mathrm{p}<$ $0.001 ; \mathrm{r}=-0.40, \mathrm{p}<0.005 ; \mathrm{r}=0.43, \mathrm{p}<0.005)$. Among the different JIA groups, patients with oligoarticular onset treated with MTX and a low dose of glucocorticoids showed a higher spine BMAD SDS than those receiving glucocorticoids alone $(\mathrm{p}<0.05)$.

Our data on the age at puberty onset showed a significant delay (years, 95\% CI) both in girls [B2 10.7 (9.6-13.0), B3 11.4 (10.4-13.6), and B4 $12.2(11.1-14.2)]$ and in boys [G2 12.3 (11.2-12.8), G3 $13.3(12.7-14.2)$, and G4 14.4 (14.0-15.5)], compared to the healthy Italian population.

The longitudinal evaluation of DEXA showed that spine BMAD SDS did not improve significantly at the second and third assessment in the whole group of patients with JIA in comparison to controls, except for those with systemic onset (Table 2 and Figures 2 and 3).

Among the JIA groups, spine BMAD SDS still correlated significantly with the type of onset. In particular, patients with systemic arthritis had lower spine BMAD SDS for oligoarticular ( $p<0.05$ at second and third DEXA), polyarticular ( $p<0.05$ at second DEXA), and enthesitis-related arthritis ( $<<0.005$ at second, and $p<0.05$ at third DEXA) subgroups, while the comparisons among the subgroups were not statistically significant.

These results were especially consistent in young adult patients in all JIA subtypes who did not develop an increase in spine BMAD during and after puberty, as is expected in healthy subjects. In particular, at the second DEXA, patients with oligoarticular onset showed a spine BMAD value of $-0.52 \pm 0.8$, and at the third $-0.46 \pm 0.9$ (vs $-0.41 \pm 0.7$, p = NS). Patients with polyarticular onset showed a spine BMAD value of $-0.60 \pm 0.8$ at the second DEXA, and -0.62 \pm 0.7 at the third (vs $-0.63 \pm 0.8, \mathrm{p}=\mathrm{NS}$ ). Patients with systemic onset showed a spine BMAD value of $-1.19 \pm 0.5$ at the second DEXA, and $-1.11 \pm 0.8$ at the third (vs $-1.41 \pm$ $0.4 ; \mathrm{p}<0.01$ and $\mathrm{p}<0.05$, respectively). Patients with enthesitis-related arthritis showed a spine BMAD value of $-0.1 \pm 0.8$ at the second DEXA, and $-0.2 \pm 0.9$ at the third (vs $-0.09 \pm 0.1, \mathrm{p}=\mathrm{NS}$ ).

Analyzing the different medication exposures longitudinally, $40 \%$ of our patients with JIA at the second DEXA and $38 \%$ at the third took NSAID ( $\mathrm{p}=\mathrm{NS}$ vs first DEXA). MTX was prescribed to $46.1 \%$ at the second DEXA, and $44.9 \%$ at the third ( $\mathrm{p}=\mathrm{NS}$ vs first DEXA). In addition, TNF- $\alpha$ inhibitor was prescribed to $19.1 \%$ at the second DEXA and $22.5 \%$ at the third DEXA ( $\mathrm{p}=\mathrm{NS}$ vs first DEXA). Finally, 


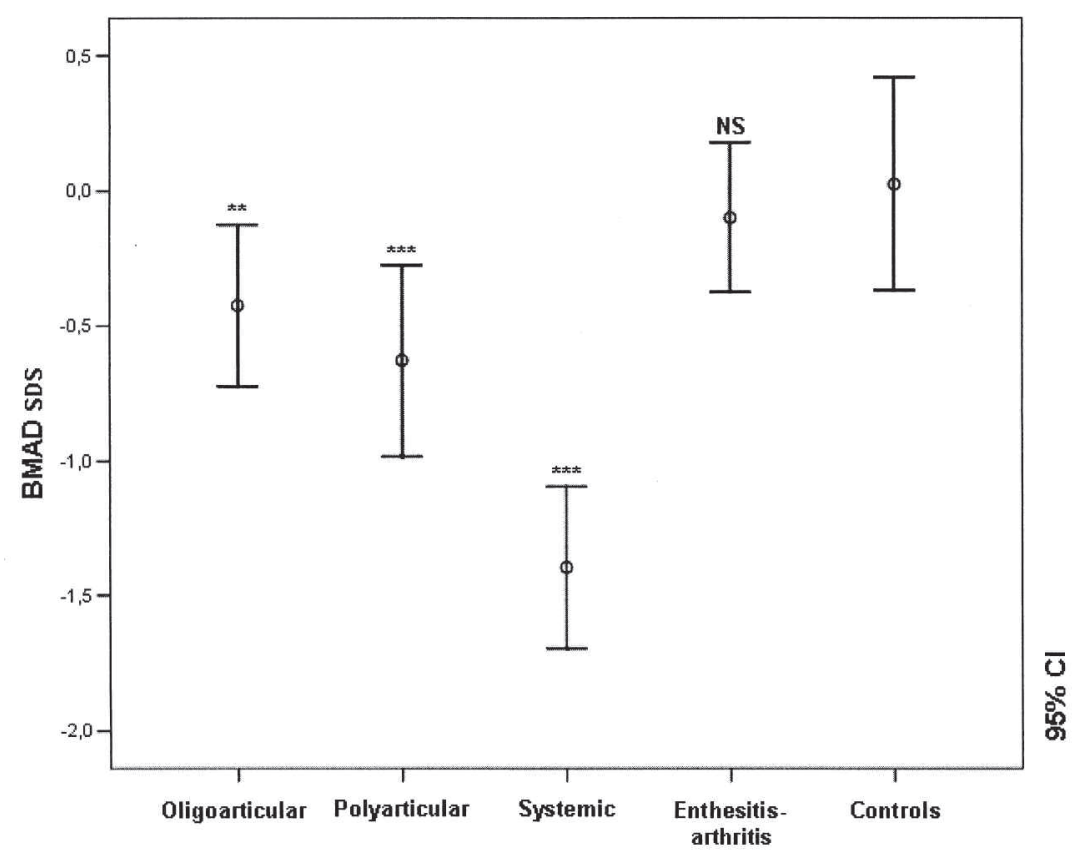

Figure 1. Spine bone mineral apparent density standard deviation scores (BMAD SDS) in patients with oligoarticular, polyarticular, systemic, and enthesitis-arthritis JIA vs controls at first DEXA scan. Bars represent mean and 95\% CI. $* *$ p $<0.005 ; * * * p<0.001$. NS: nonsignificant.

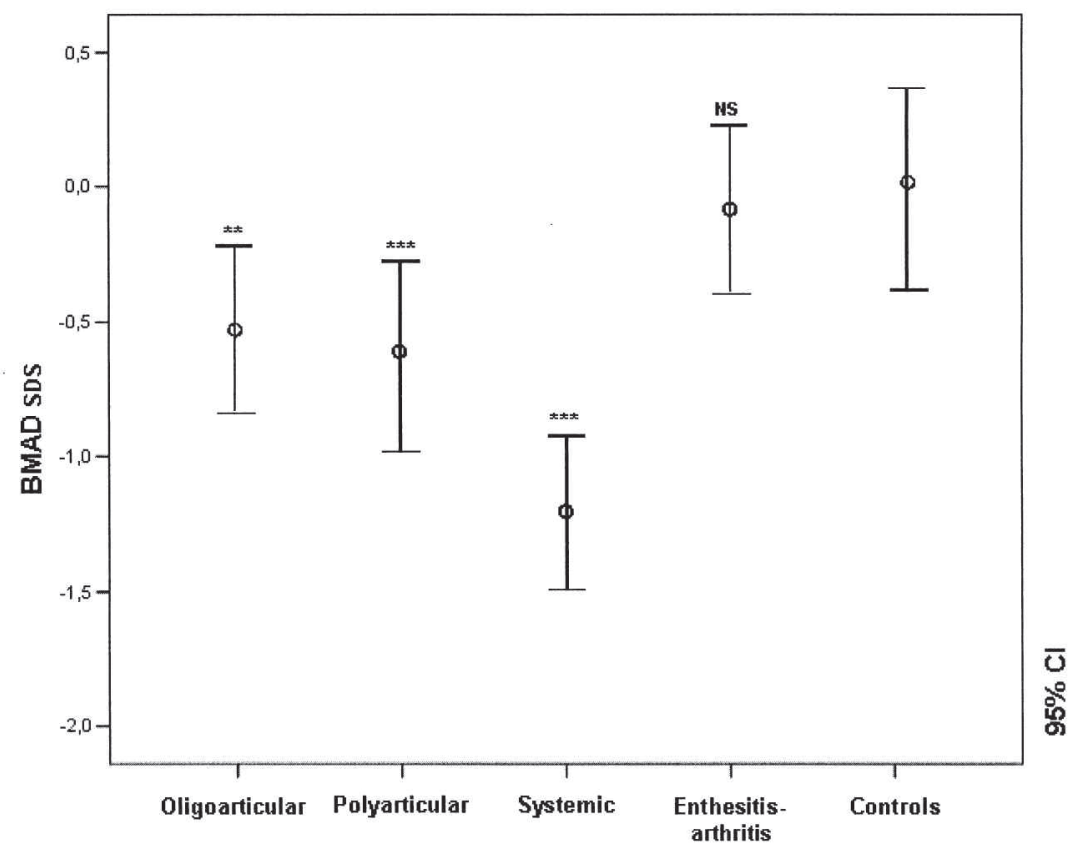

Figure 2. Spine bone mineral apparent density standard deviation scores (BMAD SDS) in patients with oligoarticular, polyarticular, systemic, and enthesitis-arthritis JIA vs controls at second DEXA scan. Bars represent mean and 95\% CI. **p $<0.005 ; * * * p<0.001$. NS: nonsignificant.

$34.8 \%$ at the second DEXA and $37.1 \%$ at the third $(\mathrm{p}=\mathrm{NS}$ vs first DEXA) took sulfasalazine. Longitudinally evaluated, high-dose systemic or oral glucocorticoid exposures were statistically significant in patients with systemic JIA onset $(\mathrm{p}<0.001)$ compared to other JIA subtypes, while the dose administered to patients with the other subtypes of the disease was without statistical difference.

In the longitudinal JIA sample, spine BMAD correlated 


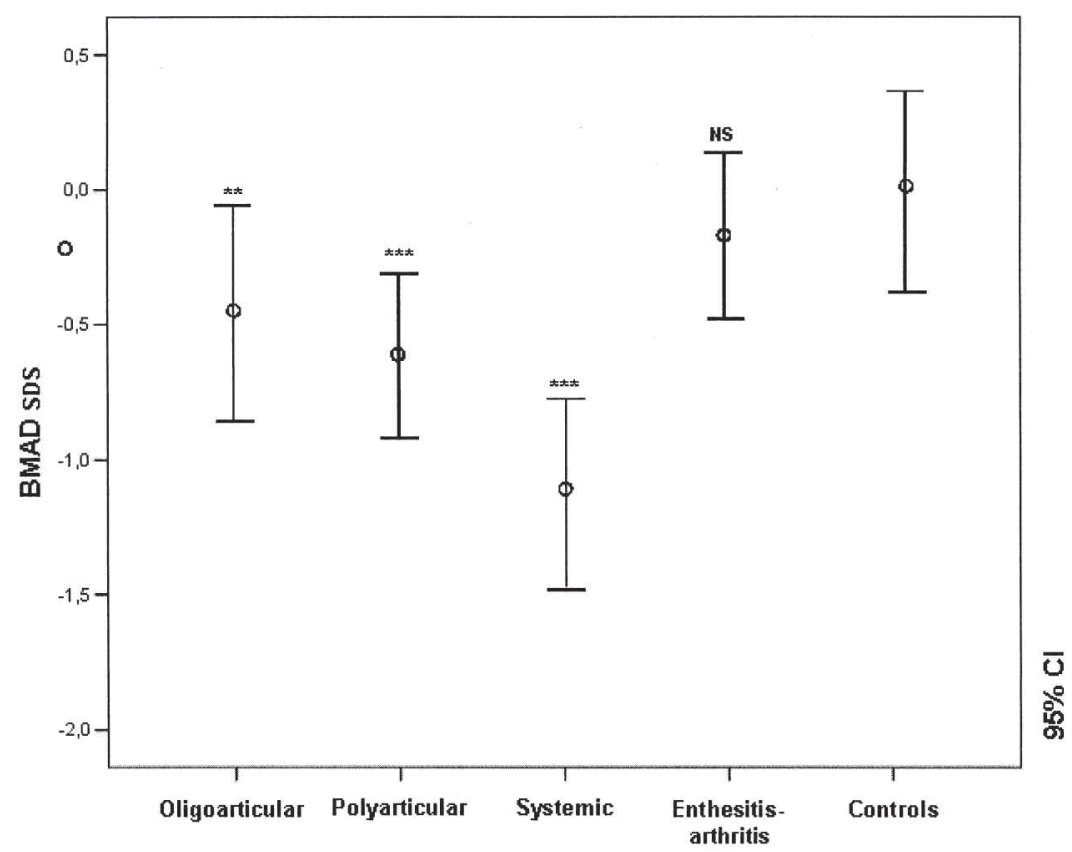

Figure 3. Spine bone mineral apparent density standard deviation scores (BMAD SDS) in patients with oligoarticular, polyarticular, systemic, and enthesitis-arthritis JIA vs controls at third DEXA scan. Bars represent mean and 95\% CI. **p $<0.005$; *** $<<0.001$. NS: nonsignificant.

with sex $(\mathrm{r}=-0.30 ; \mathrm{p}<0.01$ at the second DEXA and $\mathrm{r}=$ $-0.32 ; \mathrm{p}<0.01$ at the third), systemic corticosteroid exposure $(r=-0.40 ; p<0.01$ at the second DEXA and $r=-0.42$; $\mathrm{p}<0.01$ at the third), the number of intraarticular corticosteroid injections $(\mathrm{r}=-0.35 ; \mathrm{p}<0.01$ at the second DEXA and $r=-0.36 ; p<0.01$ at the third), the interval from last steroid injection $(\mathrm{r}=-0.41 ; \mathrm{p}<0.05$ at the second DEXA and $\mathrm{r}=-0.43 ; \mathrm{p}<0.05$ at the third $)$, ESR $(\mathrm{r}=-0.42 ; \mathrm{p}<$ 0.001 at the second DEXA and $\mathrm{r}=-0.47 ; \mathrm{p}<0.005$ at the third), and CRP levels $(r=-0.47 ; \mathrm{p}<0.001$ at the second DEXA and $r=-0.43 ; p<0.005$ at the third).

The number of flares still correlated significantly with BMI SDS $(r=-0.36 ; p<0.05)$ and height SDS $(r=-0.34$; $\mathrm{p}<0.05)$, as well as disease activity and pubertal onset $(\mathrm{r}=$ $0.37 ; \mathrm{p}<0.05)$.

Systemic corticosteroid exposure or the number of intraarticular corticosteroid injections still revealed a significant correlation with hemoglobin (respectively: $r=-0.32$, $\mathrm{p}<0.05$, at second DEXA and $\mathrm{r}=-0.34, \mathrm{p}<0.05$, at third DEXA; $r=-0.28, p<0.05$, at second DEXA and $r=-0.31$, $\mathrm{p}<0.05$, at third DEXA), ESR (respectively: $\mathrm{r}=-0.31, \mathrm{p}<$ 0.05 , at second DEXA and $\mathrm{r}=-0.33, \mathrm{p}<0.05$, at third DEXA; $r=0.30, p<0.05$, at both second and third DEXA), CRP (respectively: $r=-0.30, p<0.05$, at second DEXA and $\mathrm{r}=-0.29, \mathrm{p}<0.05$, at third DEXA; $\mathrm{r}=0.31, \mathrm{p}<0.05$, at second and $\mathrm{r}=0.33, \mathrm{p}<0.05$, at third DEXA), and pubertal stage $(r=-0.36, p<0.05$, and $r=-0.34, p<0.05$, at the third DEXA).

TNF- $\alpha$ inhibitor therapy showed a significant correlation with hemoglobin $(\mathrm{r}=-0.32, \mathrm{p}<0.01$, at second DEXA and $r=-0.30, p<0.05$, at third DEXA), ESR $(r=0.26, p<0.05$, at both second and third DEXA), CRP $(r=0.33, p<0.05$, at second and $r=0.35, p<0.01$, at third DEXA), and pubertal stage $(r=-0.36, p<0.05)$.

Finally, spine BMAD SDS did not correlate in the longitudinal evaluation with the different drugs in any JIA subtypes except systemic onset, with systemic corticosteroid exposure $(\mathrm{r}=-0.42, \mathrm{p}<0.005)$.

\section{DISCUSSION}

In the last decades the new antirheumatic drugs such as MTX and TNF- $\alpha$ inhibitors have completely changed the course and the outcome of JIA ${ }^{14}$. While several studies have reported a high prevalence of osteopenia and osteoporosis in patients with JIA ${ }^{1}$, our results suggest that the current therapies may have improved the overall skeletal health, even if an inadequate bone mass compared to controls still persists in nearly all disease subgroups, and a minority of patients develop a significant osteopenia and osteoporosis.

In previous cross-sectional studies, in which the use of DEXA was predominant, all subgroups of JIA revealed a significant decrease of bone mass at the spine and peripheral skeleton ${ }^{2,7}$. As bone mass may also be reduced by growth retardation, a common event in JIA, a correction of the results for body height is required; however, in the majority of these studies the correction was not performed ${ }^{1}$. Other risk factors for low bone mass in JIA include chronic inflammation, delayed pubertal maturation, malnutrition, 
muscle weakness, physical inactivity, and glucocorticoid therapy ${ }^{1,34}$.

The influence of childhood arthritis on bone health may be immediate, leading to fractures in childhood, or it may be delayed, resulting from suboptimal peak bone mass attainment in young adults 1,34 .

Our data, as in other studies, report a higher bone loss in systemic JIA than in oligoarticular and polyarticular onset disease. Conversely, patients with enthesitis-related arthritis displayed a bone density comparable to controls.

According to several authors, $75 \%$ of the variation in bone mass could be explained by reduced muscle mass ${ }^{3,35}$. This hypothesis is supported by the fact that $33 \%$ of patients did not have active arthritis at the time of DEXA evalua$\operatorname{tion}^{3,35}$. These results are explained in part by the effect of a reduced physical activity and/or the reduction of muscle mass that, in association with the pubertal delay, may be crucial in determining the missed enhancement of bone mass ${ }^{20}$. Investigators hypothesized that because muscle and bone deficits in JIA are highly correlated, interventions to improve muscle mass and strength will optimize bone health ${ }^{20}$.

On the other hand, our data demonstrate that children and adolescents with enthesitis-related arthritis do not have significant reduction of bone mass compared to controls; but these patients may have lower levels of physical activity, poorer physical health, and more bodily pain compared with patients with oligoarthritis or polyarthritis, and healthy controls ${ }^{36}$. Thus, reduced physical activity and/or reduction of muscle mass may not completely explain these differences between patients with JIA and controls and between patients with enthesitis-related arthritis and other JIA groups. It is possible that reduced physical activity may be important in conditioning the missed or reduced bone accrual, while the reduction of BMAD, detected frequently at the diagnosis, may be better related to inflammation.

Scarce data are reported regarding patients with enthesitis-related arthritis. Burnham, et $a l^{20}$, using peripheral quantitative computed tomography of the left tibia, reported reduced trabecular vertebral BMD Z scores in this subtype of JIA compared to controls. The observation that loss of bone mass in ankylosing spondylitis seems to occur only in patients with persistent active disease strongly suggests that inflammatory activity of the disease itself plays a major role in the pathophysiology of the early bone mineral disorders observed in these patients ${ }^{37}$. This could also depend on disease duration.

Previous cross-sectional studies showed that glucocorticoid treatment leads to a decrease in bone mass at the spine ${ }^{4,38}$, partly explaining the decrease in body height frequent in patients with JIA ${ }^{39}$. A concern of all pediatric studies is the fairly low number of patients and the heterogeneity of the amount and duration of glucocorticoid treatment ${ }^{4}$. Glucocorticoids can cause growth retardation that might lead to false low results if not corrected ${ }^{4}$. However, many studies have demonstrated that inadequate bone mineralization is almost universal in children with JIA, even if they are not taking glucocorticoids ${ }^{34}$. In agreement with other authors ${ }^{5}$, we did not discover any correlation between the total steroid dosage and BMAD values in the steroid group.

Our data indicate that notwithstanding the new therapeutic approaches, the effects of glucocorticoids are still important in conditioning negative bone accrual in these patients. However, with the advent of TNF- $\alpha$ inhibitors, a greater number of children with JIA achieve clinical remission and, potentially, normalization of muscle and bone deficits ${ }^{20}$. Our results also show that TNF- $\alpha$ inhibitor therapy is important in the early phase of JIA to improve but probably not normalize bone mass. So, other factors seem crucial in reaching a normal bone density and an appropriate bone mass peak in patients with JIA.

Indeed, on the basis of our data, BMAD seems not to improve longitudinally in patients with JIA, except for the systemic subgroup. In this last group, steroids were progressively tapered and withdrawn as systemic features subsided. MTX alone or in association with TNF- $\alpha$ inhibitor was then introduced to control joint inflammation, thus increasing bone density.

However, we also observed that MTX treatment associated with glucocorticoids in JIA with oligoarticular onset induced a less detrimental effect on bone density than just corticosteroids alone. These data are not confirmed in the other subtypes of JIA, and are in contrast with studies that showed a decrease in bone density from low-dose MTX treatment ${ }^{40}$. But these data are in agreement with other studies that do not report association between low-dose MTX and change of BMD at vertebral bone in patients with JIA ${ }^{15}$. In a study of patients treated with prednisone, the combined treatment with MTX was associated with greater bone loss at the lumbar spine ${ }^{41}$.

Our data show a critical negative role of disease activity in the regular pubertal onset, and probably in achieving an optimal bone density. The peak bone mass attained at the end of adolescent growth is critical in determining the risk of osteoporosis in adult life ${ }^{34}$. Decreased bone mass is commonly found in children and adolescents with JIA and has been observed at the onset of the disease, throughout childhood, and into adulthood, thus increasing the risk of osteoporosis and fracture later in life $\mathrm{e}^{42,43}$.

Nevertheless, reduced values of BMAD were detected in our patients, supporting the potential risk of chronic disease from lowering the accrual of normal bone. In addition, our longitudinal evaluation seems to suggest that no significant improvement in BMAD is attained in most patients with JIA under treatment at the followup. These data prove that an impaired bone mass remains a concern in children, adolescents, and young adults with JIA, even if there is no difference between patients and controls with regard to calcium or 
vitamin D intake or vitamin D and calcium serum levels ${ }^{4}$. However, calcium supplementation resulted in a small but statistically significant increase in BMD compared with placebo in children with $\mathrm{JIA}^{44}$.

Therefore, close monitoring of bone health is recommended in all children with JIA; early diagnosis of bone loss and timely identification of patients at risk for osteoporosis are essential for the prevention and management of this condition and its consequences. Preventive measures such as optimizing vitamin D and calcium intake, and weight-bearing physical activity, are also recommended.

Patients with JIA have a low bone mass and, after a first increase due to the appropriate therapy, do not reach the normal condition over time despite use of the current more effective drugs. There is a high risk of osteoporosis in early adulthood. To reduce the risk, close monitoring of BMD, better control of disease activity, physical activity, and a dietary intake of calcium and vitamin D are advised to improve bone mass. In patients with proven osteoporosis, therapeutic approaches including bisphosphonates should be considered.

\section{REFERENCES}

1. Roth J, Bechtold S, Borte G, Dressler F, Girschick HJ, Borte M. Osteoporosis in juvenile idiopathic arthritis - a practical approach to diagnosis and therapy. Eur J Pediatr 2007;166:775-84.

2. Pepmueller PH, Cassidy JT, Allen SH, Hillman LS. Bone mineralization and bone mineral metabolism in children with juvenile rheumatoid arthritis. Arthritis Rheum 1996;39:746-57.

3. Henderson CJ, Cawkwell GD, Specker BL, Sierra RI, Wilmott RW, Campaigne BN, et al. Predictors of total body bone mineral density in non-corticosteroid-treated prepubertal children with juvenile rheumatoid arthritis. Arthritis Rheum 1997;40:1967-75.

4. Falcini F, Trapani S, Civinini R, Capone A, Ermini M, Bartolozzi $\mathrm{G}$. The primary role of steroids on the osteoporosis in juvenile rheumatoid arthritis patients evaluated by dual energy X-ray absorptiometry. J Endocrinol Invest 1996;19:165-9.

5. Celiker R, Bal S, Bakkaloglu A, Ozaydin E, Coskun T, Cetin A, et al. Factors playing a role in the development of decreased bone mineral density in juvenile chronic arthritis. Rheumatol Int 2003;23:127-9.

6. McDonagh JE. Osteoporosis in juvenile idiopathic arthritis. Curr Opin Rheumatol 2001;13:399-404.

7. Roth J, Linge M, Tzaribachev N, Schweizer R, Kuemmerle-Deschner J. Musculoskeletal abnormalities in juvenile idiopathic arthritis - a 4-year longitudinal study. Rheumatology 2007;46:1180-4.

8. Strand V, Kavanaugh AF. The role of interleukin-1 in bone resorption in rheumatoid arthritis. Rheumatology 2004;43 Suppl 3:10-6.

9. Marzo-Ortega H, McGonagle D, Haugeberg G, Green MJ, Stewart $\mathrm{S}$, Emery P. Bone mineral density improvement in spondyloarthropathy after treatment with etanercept. Ann Rheum Dis 2003;62:1020-1.

10. Gravallese EM. Bone destruction in arthritis. Ann Rheum Dis 2002;61:84-6.

11. Lindehammar H, Lindvall B. Muscle involvement in juvenile idiopathic arthritis. Rheumatology 2004;43:1546-54.

12. Häkkinen A, Sokka T, Kautiainen H, Kotaniemi A, Hannonen P. Sustained maintenance of exercise induced muscle strength gains and normal bone mineral density in patients with early rheumatoid arthritis: a 5 year follow up. Ann Rheum Dis 2004;63:910-6.

13. Falcini F, Bindi G, Ermini M, Galluzzi F, Poggi G, Rossi S, et al. Comparison of quantitative calcaneal ultrasound and dual energy $\mathrm{X}$-ray absorptiometry in the evaluation of osteoporotic risk in children with chronic rheumatic diseases. Calcif Tissue Int 2000;67:19-23

14. Haines KA. Juvenile idiopathic arthritis: therapies in the $21 \mathrm{st}$ century. Bull NYU Hosp Jt Dis 2007;65:205-11.

15. Bianchi ML, Cimaz R, Galbiati E, Corona F, Cherubini R, Bardare $\mathrm{M}$. Bone mass change during methotrexate treatment in patients with juvenile rheumatoid arthritis. Osteoporos Int 1999;10:20-5.

16. Simonini G, Giani T, Stagi S, de Martino M, Falcini F. Bone status over $1 \mathrm{yr}$ of etanercept treatment in juvenile idiopathic arthritis. Rheumatology 2005;44:777-80.

17. Henderson CJ, Panush RS. Diets, dietary supplements, and nutritional therapies in rheumatic diseases. Rheum Dis Clin North Am 1999;25:937-68, ix.

18. Varonos S, Ansell BM, Reeve J. Vertebral collapse in juvenile chronic arthritis: its relationship with glucocorticoid therapy. Calcif Tissue Int 1987;41:75-8.

19. Lien G, Flatø B, Haugen M, Vinje O, Sørskaar D, Dale K, et al. Frequency of osteopenia in adolescents with early-onset juvenile idiopathic arthritis: a long-term outcome study of one hundred five patients. Arthritis Rheum 2003;48:2214-23.

20. Burnham JM, Shults J, Dubner SE, Sembhi H, Zemel BS, Leonard MB. Bone density, structure, and strength in juvenile idiopathic arthritis: importance of disease severity and muscle deficits. Arthritis Rheum 2008;58:2518-27.

21. Górska A, Urban M, Bartnicka M, Zelazowska-Rutkowska B, Wysocka J. Bone mineral metabolism in children with juvenile idiopathic arthritis - preliminary report. Ortop Traumatol Rehabil 2008;10:54-62.

22. Pereira RM, Corrente JE, Chahade WH, Yoshinari NH. Evaluation by dual X-ray absorptiometry (DXA) of bone mineral density in children with juvenile chronic arthritis. Clin Exp Rheumatol 1998;16:495-501.

23. Kotaniemi A, Savolainen A, Kröger H, Kautiainen H, Isomäki H. Weight-bearing physical activity, calcium intake, systemic glucocorticoids, chronic inflammation, and body constitution as determinants of lumbar and femoral bone mineral in juvenile chronic arthritis. Scand J Rheumatol 1999;28:19-26.

24. Lurati A, Cimaz R, Gattinara M, Gerloni V, Teruzzi B, Salmaso A, et al. [Skeletal mineralization in a prepubertal female population affected by juvenile idiopathic arthritis]. Reumatismo 2008; 60:224-9.

25. Lien G, Selvaag AM, Flatø B, Haugen M, Vinje O, Sørskaar D, et al. A two-year prospective controlled study of bone mass and bone turnover in children with early juvenile idiopathic arthritis. Arthritis Rheum 2005;52:833-40.

26. Petty RE, Southwood TR, Manners P, Baum J, Glass DN, Goldenberg J, et al. International League of Associations for Rheumatology. International League of Associations for Rheumatology classification of juvenile idiopathic arthritis: second revision, Edmonton, 2001. J Rheumatol 2004;31:390-2.

27. Cacciari E, Milani S, Balsamo A, Spada E, Bona G, Cavallo L, et al. Italian cross-sectional growth charts for height, weight and BMI ( 2 to 20 yr). J Endocrinol Invest 2006;29:581-93.

28. Tanner JM, Whitehouse RH. Clinical longitudinal standards for height, weight, height velocity, weight velocity, and stages of puberty. Arch Dis Child 1976;51:170-9.

29. Stagi S, Galli L, Cecchi C, Chiappini E, Losi S, Gattinara CG, et al. Final height in patients perinatally infected with the human immunodeficiency virus. Horm Res 2010; [in press].

30. Kröger H, Kotaniemi A, Vainio P, Alhava E. Bone densitometry of 
the spine and femur in children by dual-energy $\mathrm{x}$-ray absorptiometry. Bone Miner 1992;17:75-85.

31. Kröger H, Vainio P, Nieminen J, Kotaniemi A. Comparison of different models for interpreting bone mineral density measurements using DXA and MRI technology. Bone 1995; 17:157-9.

32. Giannini EH, Ruperto N, Ravelli A, Lovell DJ, Felson DT, Martini A. Preliminary definition of improvement in juvenile arthritis. Arthritis Rheum 1997;40:1202-9.

33. Brunner HI, Lovell DJ, Finck BK, Giannini EH. Preliminary definition of disease flare in juvenile rheumatoid arthritis. J Rheumatol 2002;29:1058-64.

34. Cimaz R. Osteoporosis in childhood rheumatic diseases: prevention and therapy. Best Pract Res Clin Rheumatol 2002;16:397-409.

35. Henderson CJ, Specker BL, Sierra RI, Campaigne BN, Lovell DJ. Total-body bone mineral content in non-corticosteroid-treated postpubertal females with juvenile rheumatoid arthritis: frequency of osteopenia and contributing factors. Arthritis Rheum 2000;43:531-40.

36. Flat $\varnothing$ B, Hoffmann-Vold AM, Reiff A, Førre $\varnothing$, Lien G, Vinje O. Long-term outcome and prognostic factors in enthesitis-related arthritis: a case-control study. Arthritis Rheum 2006;54:3573-82.

37. Gratacós J, Collado A, Pons F, Osaba M, Sanmartí R, Roqué M, et al. Significant loss of bone mass in patients with early, active ankylosing spondylitis: a followup study. Arthritis Rheum 1999;42:2319-24.
38. Habib GS. Systemic effects of intra-articular corticosteroids. Clin Rheumatol 2009;28:749-56.

39. Kotaniemi A, Savolainen A, Kautiainen H, Kroeger H. Estimation of central osteopenia in children with chronic polyarthritis treated with glucocorticoids. Pediatrics 1993;91:1127-30.

40. Hartman C, Shamir R, Eshach-Adiv O, Iosilevsky G, Brik R. Assessment of osteoporosis by quantitative ultrasound versus dual energy X-ray absorptiometry in children with chronic rheumatic diseases. J Rheumatol 2004;31:981-5.

41. Habib GS, Haj S. Bone mineral density in patients with early rheumatoid arthritis treated with corticosteroids. Clin Rheumatol 2005;24:129-33.

42. Cetin A, Celiker R, Dincer F, Ariyurek M. Bone mineral density in children with juvenile chronic arthritis. Clin Rheumatol 1998; 17:551-3.

43. Aggarwal P, Aggarwal A, Gupta S, Misra R. Osteopenia is common in adult male patients with active juvenile idiopathic arthritis. J Rheumatol 2006;33:1642-5.

44. Lovell DJ, Glass D, Ranz J, Kramer S, Huang B, Sierra RI, et al. A randomized controlled trial of calcium supplementation to increase bone mineral density in children with juvenile rheumatoid arthritis Arthritis Rheum 2006;54:2235-42. 